\title{
Diffusion imaging and tractography of congenital brain malformations
}

\author{
Michael Wahl • A. James Barkovich • Pratik Mukherjee
}

Received: 8 July 2009 /Revised: 7 October 2009 /Accepted: 19 October 2009 / Published online: 24 November 2009

(C) The Author(s) 2009. This article is published with open access at Springerlink.com

\begin{abstract}
Diffusion imaging is an MRI modality that measures the microscopic molecular motion of water in order to investigate white matter microstructure. The modality has been used extensively in recent years to investigate the neuroanatomical basis of congenital brain malformations. We review the basic principles of diffusion imaging and of specific techniques, including diffusion tensor imaging (DTI) and high angular resolution diffusion imaging (HARDI). We show how DTI and HARDI, and their application to fiber tractography, has elucidated the aberrant connectivity underlying a number of congenital brain malformations. Finally, we discuss potential uses for diffusion imaging of developmental disorders in the clinical and research realms.
\end{abstract}

Keywords Diffusion tensor - DTI · White matter · Brain malformations $\cdot$ Children

\section{Introduction}

Diffusion MRI has quickly become an important part of neuroradiology research, finding application in essentially every disorder involving white matter, from schizophrenia to multiple sclerosis to traumatic brain injury. Within the field of pediatric radiology, diffusion imaging has allowed

\section{Wahl}

Department of Neurology, University of California,

San Francisco,

San Francisco, CA, USA

M. Wahl · A. J. Barkovich · P. Mukherjee $(\square)$

Department of Radiology, University of California, San Francisco,

505 Parnassus Ave., L-358, Box 0628,

San Francisco, CA 94143-0628, USA

e-mail: pratik@radmail.radiology.ucsf.edu researchers to make discoveries about the neuroanatomical features underlying several congenital disorders.

This review will briefly summarize the theory behind diffusion MR imaging and discuss newer techniques for more advanced white matter microstructural analysis and fiber tractography, including diffusion tensor imaging (DTI) and high angular resolution diffusion imaging (HARDI). We explore the application of diffusion imaging and fiber tracking to four congenital brain malformations: horizontal gaze palsy with progressive scoliosis (HGPPS), pontine tegmental cap dysplasia (PTCD), holoprosencephaly, and agenesis of the corpus callosum (AgCC). Finally we discuss potential applications of diffusion imaging in clinical neuroradiology. This discussion is not intended to be an exhaustive review of the DTI literature on congenital malformations. Rather, we show in depth how these new imaging technologies have contributed knowledge for a few conditions, in order to highlight the uses of diffusion imaging in research and clinical settings.

\section{Diffusion imaging}

Theory and acquisition

Large white matter tracts are composed of numerous axons organized in a parallel fashion. Water contained within these structures diffuses through random molecular motion (Brownian motion), but such motion is constrained by densely packed axonal membranes and myelin sheaths. Thus water will preferentially diffuse along the direction of the axon bundle rather than perpendicular to it. The measurement of this anisotropic diffusion of water forms the basis for diffusion imaging $[1,2]$.

Diffusion imaging is performed by adding a pair of diffusion-sensitizing spatial gradient pulses to a $\mathrm{T} 2$-weighted 
spin-echo MR sequence. The gradients are applied in the same direction before and after the $180^{\circ}$ refocusing RF pulse, a sequence called Stejskal-Tanner diffusion encoding [3]. Thus molecular motion of water along the spatial gradient causes an incomplete rephasing of proton spins and resultant loss of signal intensity. Whole-brain images are acquired with the gradient applied in a number of independent directions; a reference image with no applied gradient is also acquired. Information from all images can then be combined to evaluate the three-dimensional profile of diffusion in each voxel. Key acquisition parameters include the $\mathrm{b}$ factor, which depends on the strength, duration, and timing of gradient pulses, and the number of diffusion-encoding directions acquired. Typical $b$ values used in the clinical setting range from $600 \mathrm{~s} / \mathrm{mm}^{2}$ to $1,500 \mathrm{~s} / \mathrm{mm}^{2}$, though high angular resolution techniques (discussed below) use higher values [4].

\section{Diffusion tensor imaging}

Diffusion tensor imaging approximates the diffusion profile in each voxel as a three-dimensional Gaussian function, most commonly visualized as an ellipsoid. Mathematically, this approximation is represented by a symmetric $3 \times 3$ matrix with six independent elements (the "diffusion tensor") [1]. DTI thus requires diffusion measurements in at least six non-collinear directions, although more are frequently used to improve image quality. From the diffusion tensor, a number of useful calculations can be performed. First, the three eigenvectors and eigenvalues of the matrix can be calculated. These correspond to the directions and lengths of the three principal axes of the ellipsoid. The eigenvector corresponding to the largest eigenvalue represents the major axis, and thus (in brain imaging) the predominant direction of axons within a given voxel.

Also, the proportion of diffusion along the major axis relative to the minor axes is calculated as the fractional anisotropy (FA) and represents a quantitative measure of white matter microstructural integrity. While the precise biological factors that contribute to FA are unclear, it is thought of as a composite measure of axonal density, directional coherence of axon bundles, and degree of myelination [5]. FA values take a range from 0 (representing isotropic diffusion) to 1 (representing diffusion entirely restricted to one direction). FA has been found to be a highly reproducible measurement in healthy subjects [6] and has been shown to correlate with cognitive performance on a variety of measures in both adult [7-9] and pediatric populations [10-12].

The direction of the primary eigenvector is commonly combined with FA measurements on DTI colormaps, in which the color of each voxel represents the principal diffusion direction, and the brightness represents FA. These colormaps provide a visual representation of the size and orientation of various white-matter structures and are useful for both diagnostic and research purposes. DTI tractography can also be performed in which directional information from neighboring voxels is combined to estimate the threedimensional structure of major white-matter pathways [1315]. In congenital malformations, tractography can be used to characterize dysplastic white-matter tracts or to identify ectopic fibers. In addition, FA measurements can be averaged over an entire white-matter tract, yielding a composite quantitative measure of tract organization. A recent two-part review of DTI provides additional details on its theory and application, including recommended acquisition parameters for both $1.5-\mathrm{T}$ and 3-T systems $[4,16]$.

High angular resolution diffusion imaging

A major disadvantage of DTI is its inability to recover more than one fiber orientation within a single voxel. Thus, in regions of complex white matter architecture, where fibers merge or cross, DTI is unable to accurately depict white matter microstructure. To overcome this limitation, HARDI combines stronger diffusion-weighting (higher $b$ values) with diffusion measurements in a larger number of independent directions (on the order of 50 or more) to generate a high angular resolution measurement of threedimensional (3-D) diffusion [17]. HARDI measurements yield a more complete description of the 3-D diffusion profile than the diffusion tensor and thus facilitate the reconstruction of multiple fiber directions within a single voxel. Q-ball (QBI) imaging is one example of a HARDI reconstruction technique that has yielded detailed information about complex white matter structure within a clinically feasible scan time [18-20]. HARDI methods have not been extensively used to investigate pediatric conditions but are likely to find increasing use in the future.

\section{Applications to congenital malformations}

Horizontal gaze palsy with progressive scoliosis

Horizontal gaze palsy with progressive scoliosis (HGPPS) is a congenital malformation limited to the hindbrain and spine. It is a rare autosomal-recessive condition characterized by congenital absence of normal horizontal gaze, as well as scoliosis that progresses through childhood and adolescence. No other neurological or behavioral abnormalities have been associated with the condition. The causative gene has been identified as $\mathrm{ROBO}$, which encodes a receptor important for axon guidance across the midline [21]. Both structural and diffusion imaging have been performed on individuals with HGPPS. 
Multiple studies have reported structural imaging findings with HGPPS [22-25]. These studies report common findings of a depression of the floor of the fourth ventricle, hypoplastic pons and medulla with a "butterfly medulla" sign from an anterior median fissure, and a deep midline pontine cleft (a "split pons sign") (Fig. 1) [23]. In addition, hypoplasia of the midbrain tegmentum and cerebellar peduncles [24], and absence of the facial colliculi [23] have been noted. These studies localize the disorder to the hindbrain, provide key signs that are useful for diagnostic purposes, and give some indication of alterations to the brainstem at the midline.

DTI investigations have greatly advanced our understanding of the anatomy underlying HGPPS. One study examined the DTI color images of a patient with HGPPS to find the absence of normal decussating fibers in the pons, including superior cerebellar peduncles and pontocerebellar fibers (Fig. 1) [24]. Another study performed DTI tractography to reveal exclusively ipsilateral ascending and descending connectivity through the lower brainstem, as well as ipsilaterally projecting tracts from primary motor areas [26]. The corpus callosum was found to be grossly normal, suggesting a midline-crossing defect localized to the brainstem. This study also made functional MRI measurements while the patient performed a motor activity, which revealed only ipsilateral motor and sensory cortical activation. These DTI studies, together with the anatomic findings of deep midline clefts in the brainstem, suggest that a general defect in hindbrain midline axon crossing underlies HGPPS. These findings are in excellent agreement with the known function of the causative gene (ROBO3) in guiding midline axon crossing. Thus DTI analysis, along with genetic studies, has successfully elucidated the molecular and neuroanatomical basis of HGPPS.

\section{Pontine tegmental cap dysplasia}

The mechanisms underlying pontine tegmental cap dysplasia (PTCD) have been less fully elucidated. Clinical findings in PTCD are more varied and include multiple cranial neuropathies (acoustic nerve palsy in all patients with variable involvement of facial and trigeminal nerves), impaired swallowing, and cerebellar and pyramidal motor symptoms [27]. Global developmental delay is commonly noted, suggesting supratentorial involvement. The genetic basis for PTCD has not been found; analysis of axonal guidance genes NTN1 and DCC failed to identify any pathogenic mutations [28].

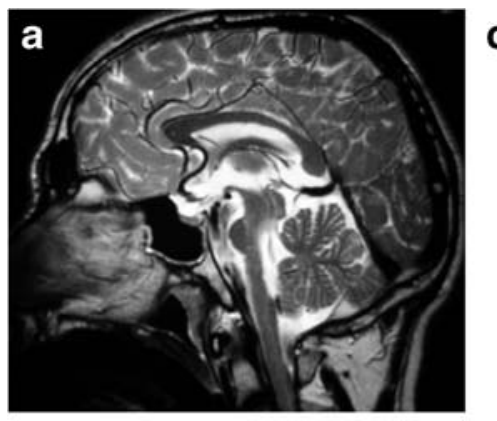

C
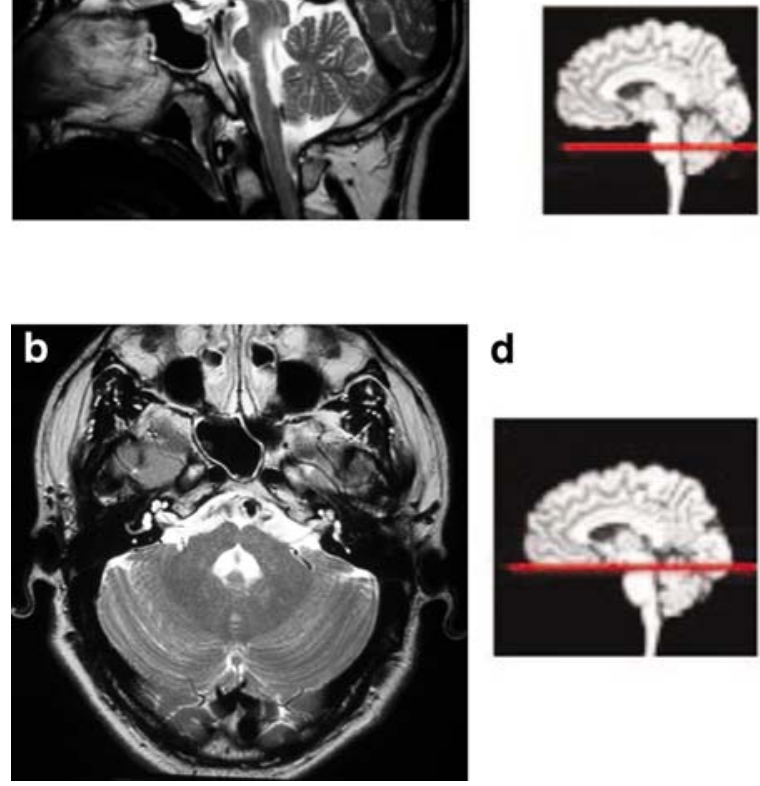

d

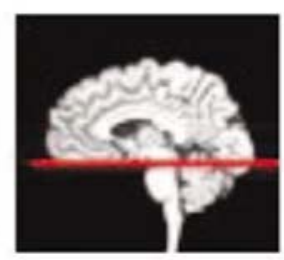

Fig. 1 Horizontal gaze palsy with progressive scoliosis. T2-W sagittal image (a) and axial image (b) of the brainstem demonstrate hypoplastic pons and medulla, with a normal callosum, and a midline cleft at the level of the pons. DTI colormaps of the middle (c) and superior (d) pons demonstrate the absence of normal decussating pontocerebellar fibers and superior cerebellar peduncles (SCPs) in
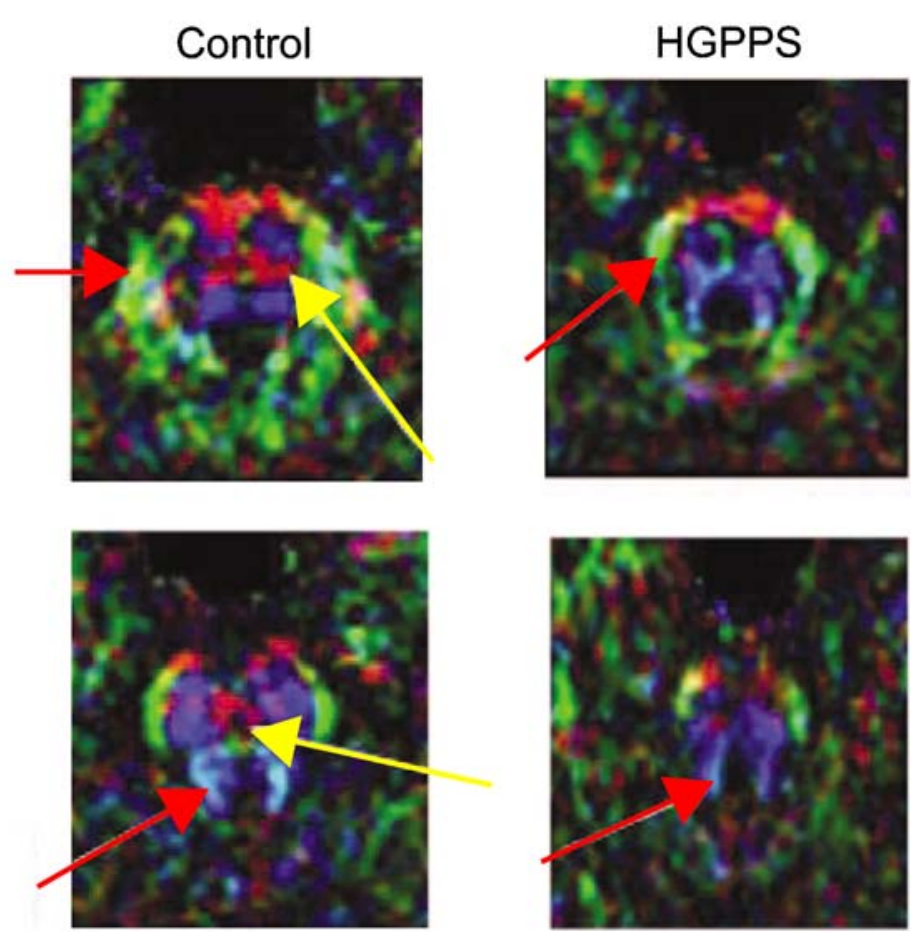

HGPPS compared to control subjects. Red arrows indicate the middle cerebellar peduncle (c) and SCP (d), while yellow arrows indicate normal decussating fibers in control subjects. For all images, color depicts the predominant fiber direction, with blue denoting superiorinferior, green denoting anterior-posterior, and red denoting left-right fiber orientation. (Figure reprinted with permission from [24]) 
Conventional MR imaging analysis reveals a number of hindbrain abnormalities. Hypoplastic ventral pons with a dorsal "cap" projecting into the fourth ventricle is the characteristic finding (Fig. 2). Other abnormalities include small cerebellar hemispheres and middle cerebellar peduncles, dysmorphic superior and inferior cerebellar peduncles, and a thickening of the isthmus at the pontomesencephalic junction. Corpus callosum dysplasia was also noted in some subjects, supporting the clinical suggestion of supratentorial involvement, although no cortical malformations were found in six patients [27]. While it is evident from conventional imaging that there is extensive hindbrain malformation, the underlying anatomical cause is unclear.

Diffusion imaging has helped point toward the pathologic basis for PTCD. Tractography studies have revealed the absence of superior and middle cerebellar peduncle decussation, as well as an ectopic fiber tract that crosses the midline on the dorsal aspect of the pons, forming the characteristic tegmental cap (Fig. 2) [27, 28]. This ectopic fiber tract finding points to a specific pathology of either neuronal migration or axon guidance of pontine gray neurons originating from the rhombic lip and has generated specific hypotheses to explain its presence (Fig. 2) [27]. Possibilities include insufficient or aberrant neuronal migration away from the rhombic lip with normal subsequent axonal guidance toward the contralateral MCP, or normal neuronal migration with aberrant axon guidance away from the ventral surface. These processes are mediated by multiple guidance molecules and suggest candidate genes that might be responsible for the condition. Although the causative gene has not been identified, DTI findings have narrowed the focus of the search.

\section{Holoprosencephaly}

Holoprosencephaly is a congenital disorder of forebrain development characterized by the partial or complete failure

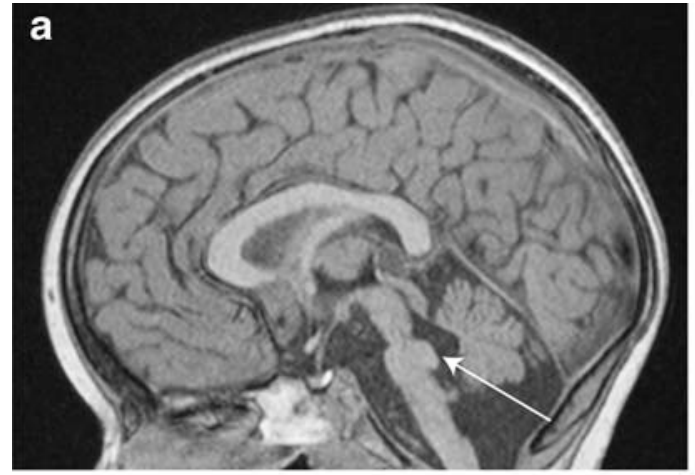

C

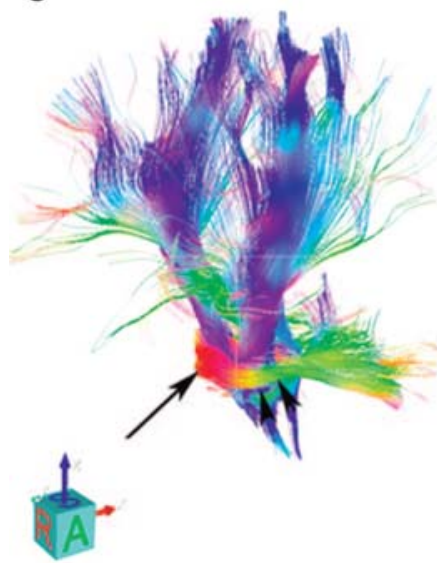

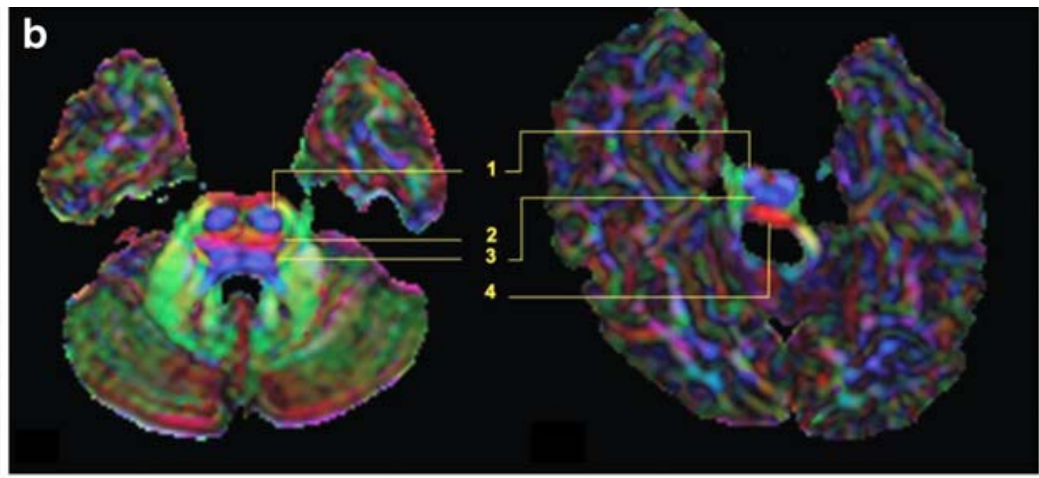

d
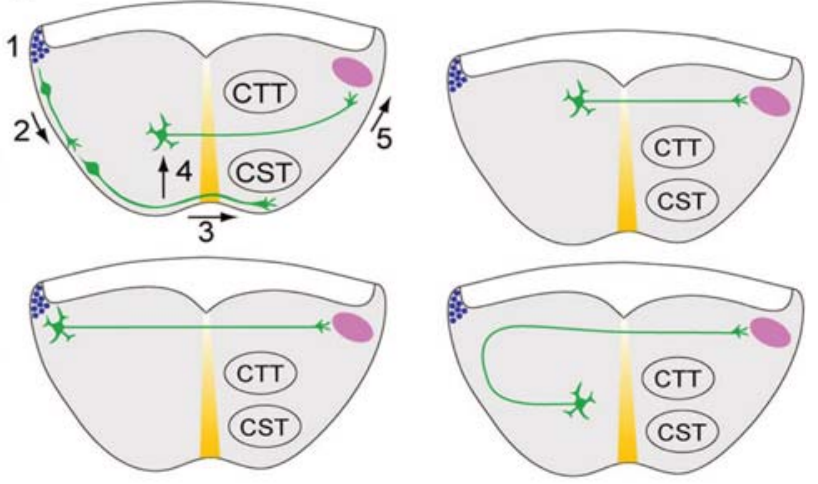

Fig. 2 Pontine tegmental cap dysplasia. a Sagittal T1-W image shows characteristic pontine cap (white arrow). b Axial DTI colormaps of control subject (left) and PTCD patient (right) show absence of normal ventral decussating pontine structures, as well as the ectopic dorsal fiber bundle. Numbers indicate pontine structures: $1=$ corticospinal tract, 2 = pontocerebellar fibers, $3=$ medial lemniscus, $4=$ ectopic transverse fiber bundle. c DTI tractography of control subject (left) and PTCD patient (right) demonstrates absence of ventral crossing fibers, and the ectopic dorsal tract. d Possible mechanisms for the formation the ectopic dorsal fiber bundle, because of either defects in axon guidance or neuronal migration of pontine gray neurons (green), produced in rhombic lip (blue circles) and sending axonal projections to the MCP (pink oval). Upper left diagram shows normal migration pattern; others demonstrate possible growth and guidance defects that could explain the neuroradiological findings. (Figure reprinted with permission from $[27,28]$ ) 
of induction of the anterior ventral forebrain with resultant absence of the anterior ventromedial cerebrum and absent anterior interhemispheric fissure. It is usually classified, from least to most severe, as lobar, semilobar or alobar. Clinical characteristics include mental retardation, facial dysmorphism, spastic quadriparesis, athetoid movements, epilepsy and endocrine disorders [29]. Conventional MR imaging studies have focused on further characterizing the condition based on morphology of the corpus callosum [30], basal ganglia [31] and sulcal patterns [29]. The last study [29] found the angle between sylvian fissures (the "sylvian angle") in the two hemispheres to be a reliable marker for severity of holoprosencephaly.

To our knowledge, only two diffusion imaging studies of holoprosencephaly have been conducted. One study performed DTI on 13 patients with holoprosencephaly, focusing on white matter tracts in the brainstem [32]. Corticospinal tracts were found to be absent bilaterally in the two people with alobar holoprosencephaly, while corticospinal tracts were recovered in all but one of the patients with less severe variants. Also, the size of corticospinal tracts and middle cerebellar peduncles were assessed from DTI color images and compared with both the type of holoprosencephaly and subject scores on neurodevelopmental exams (Fig. 3). The sizes of both tracts were found to correlate strongly with both holoprosencephaly type and neurodevelopmental score. Thus the study characterized involvement of the brainstem and provided valuable imaging markers for the severity of the condition. A report also performed DTI tractography in a case of semilobar holoprosencephaly [33]. The study found several altered white matter structures, including dysplastic fornices, fused bilateral fronto-occipital fascicule, and a posterior commissural bundle of transverse fibers in the expected location of the splenium. Further imaging of patients with all types of holoprosencephaly could provide a more complete picture of white matter dysplasia, although the relative rarity and severity of the condition make large cohort studies difficult.

\section{Agenesis of the corpus callosum}

Agenesis of the corpus callosum ( $\mathrm{AgCC})$ is a congenital brain malformation with a frequency of about 1 in 4,000 individuals. It is characterized by the partial or complete absence of callosal fibers, and accompanied by a spectrum of neuropsychological deficits, including many falling
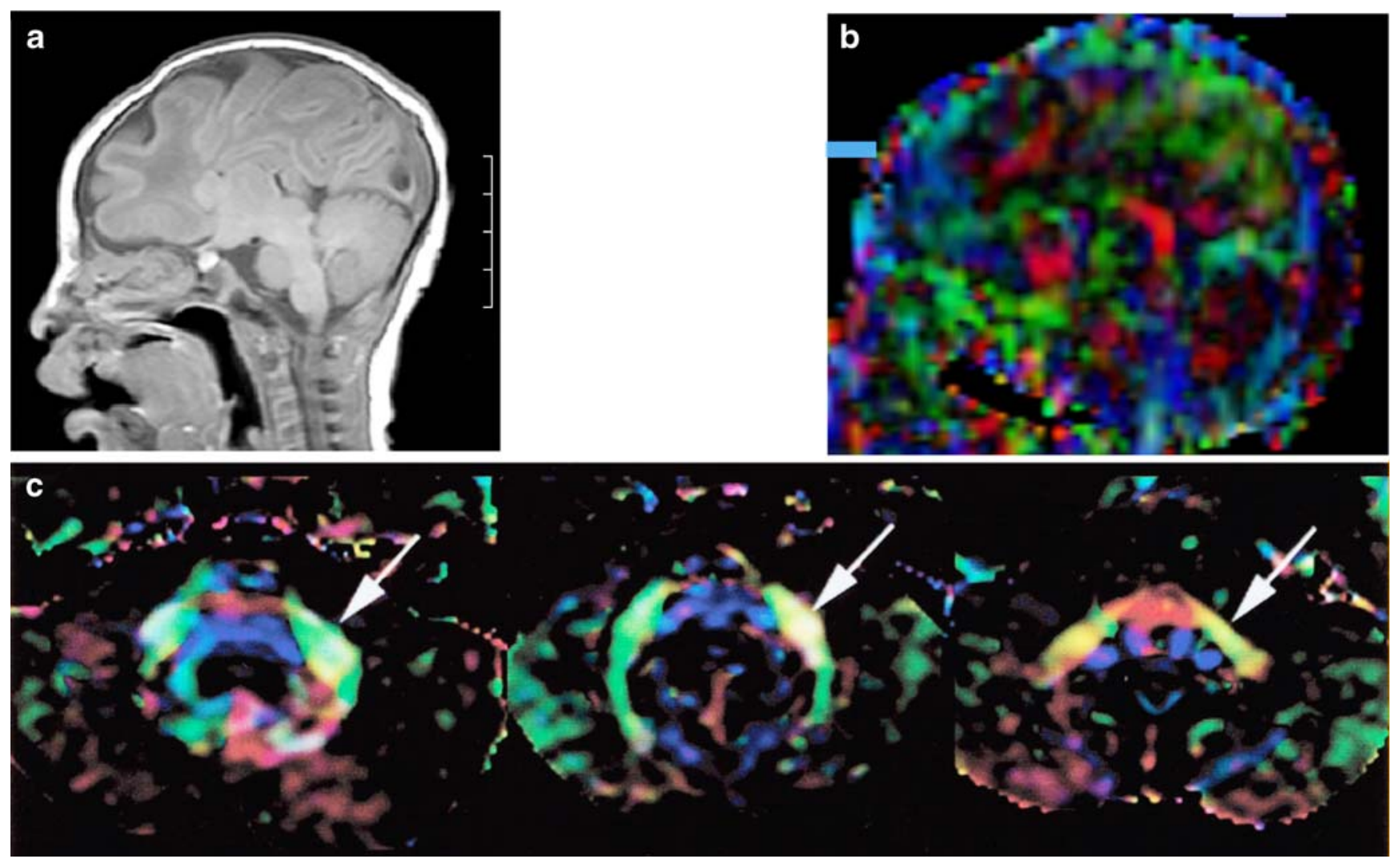

Fig. 3 Holoprosencephaly. T1-W (a) and DTI (b) mid-sagittal images of semilobar holoprosencephaly patient show abnormal sulcal patterns, fused caudate nuclei and thalami, and a transversely located white matter bundle in the expected location of the splenium. DTI imaging of middle cerebellar peduncles (c) shows progressively smaller tract size in lobar, semilobar and alobar holoprosencephaly patients (left to right, white arrows indicate MCP). (Figure reprinted with permission from $[32,33]$ ) 
within the autistic spectrum [34]. Interestingly, the symptoms associated with $\mathrm{AgCC}$ are not replicated by surgical transection of the callosum, and interhemispheric integration of information is relatively intact in most $\mathrm{AgCC}$ individuals [35]. Thus the symptoms seen with $\mathrm{AgCC}$ cannot be explained by callosal absence alone. Much imaging research into $\mathrm{AgCC}$ has focused on identifying and characterizing other possible anatomic features that contribute to the phenotype.

Studies using conventional MR imaging have focused on identifying and classifying brain malformations associated with AgCC. One study found polymicrogyria (PMG) and cortical and subcortical heterotopias to be common [36]. However, many people with $\mathrm{AgCC}$ have no associated cortical malformations yet show similar symptoms to those who do. While conventional MR imaging remains useful for diagnosing $\mathrm{AgCC}$ and cataloging other anatomic features that are evident clinically, it remains an inadequate technique for probing the basic pathobiology behind AgCC.

Diffusion imaging and tractography studies have focused on several anatomic features of $\mathrm{AgCC}$, in an effort to better understand the development of the condition. Aside from callosal absence, the most obvious anatomic feature of $\mathrm{AgCC}$ is the presence of Probst bundles, large longitudinally oriented intrahemispheric white-matter tracts running superior to the lateral ventricles (Fig. 4). These fibers are thought to be formed from misdirected callosal axons and are present in a majority of subjects with AgCC [36]. The function of the Probst bundles is unknown. Early DTI studies confirmed the anteroposterior direction of the Probst
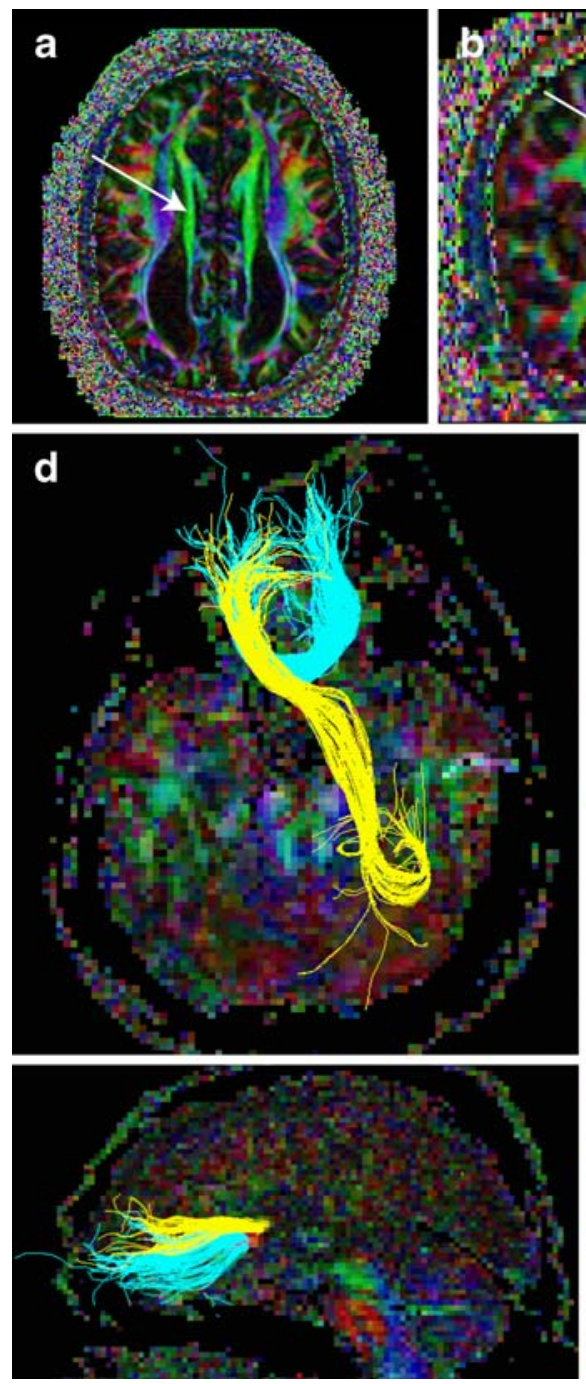
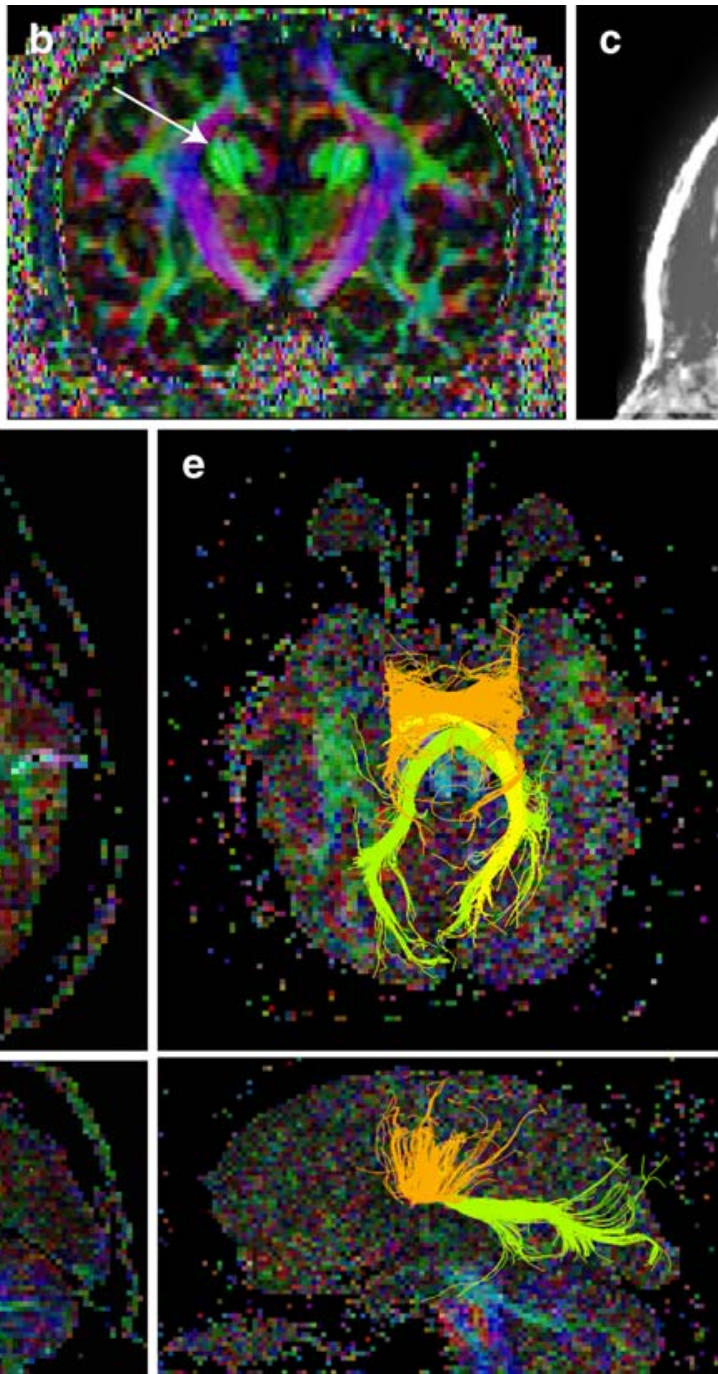
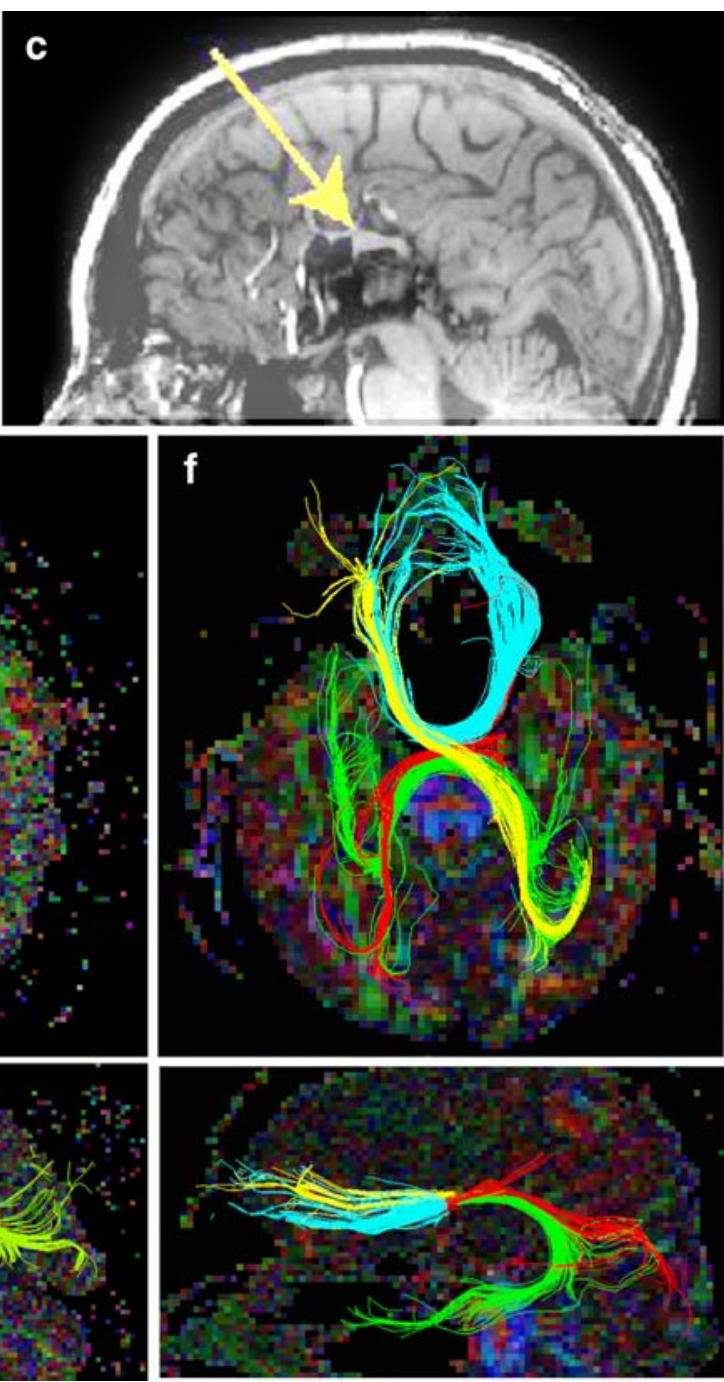

Fig. 4 Agenesis of the corpus callosum (AgCC). a, b Axial and coronal DTI colormap images show bilateral Probst bundles (arrows) in a complete AgCC patient. c T1-W mid-sagittal image of a partial AgCC (pAgCC) patient demonstrates a posteriorly located callosal fragment (yellow arrow). $\mathbf{d}-\mathbf{f}$ Q-ball tractography of three patients with pAgCC, overlaid on axial (top) and sagittal (bottom) DTI colormap images. Fiber tracts are colored according to specific cortical projections: homotopic anterior frontal, blue; homotopic posterior frontal, orange; homotopic occipito-temporal, green; heterotopic fibers, yellow and red. (Figure reprinted with permission from [42]) 
bundles and established that fibers within the bundle are at least partially topographically organized [37-39]. However, based on mouse models, the architecture of the Probst bundles is likely to be quite complex [40], making detailed tractography difficult.

Recent preliminary work using Q-ball imaging and tractography suggests that a significant number of fibers in the Probst bundles are not in fact corticocortical association fibers but instead project to subcortical regions, including thalamus and brainstem (unpublished data). This observation has an interesting correspondence in a mouse knockout model of $\mathrm{AgCC}$, in which the Satb2 gene is disrupted, causing callosal axons to be reprogrammed to project to ipsilateral subcortical regions [41]. This correspondence might point to specific candidate genes for AgCC. Whether this initial finding of aberrant subcortical connectivity in $\mathrm{AgCC}$ is verified remains to be seen, but it highlights the power of diffusion tractographic studies over conventional imaging: while conventional imaging provides useful clinical anatomic markers, diffusion imaging studies of white-matter connectivity enable investigators to probe the basic underlying developmental pathobiology that gave rise to the condition.

Additional diffusion-imaging investigations have focused on individuals with partial callosal agenesis (pAgCC). Subjects with pAgCC retain a residual callosal fragment but are symptomatically similar to those with complete AgCC [35]. There are examples of individuals with partial and complete agenesis within a single family, suggesting that the developmental and genetic causes of the two conditions are similar. Based on conventional imaging studies alone, it is unclear whether the callosal fragments are simply a result of the arrested growth of normal callosal projections or they are formed in a more complex manner.

Two diffusion imaging studies have examined the callosal connectivity in $\mathrm{pAgCC}$ in some detail. The first study used DTI tractography to examine five patients with pAgCC [39]. Within these patients, the study found a relatively consistent pattern of anteriorly located callosal fragments with primarily homotopic frontal connectivity. The study also revealed an "asymmetric sigmoid bundle" that coursed from right anterior frontal lobe to left occipitotemporal lobes. This heterotopic callosal fiber is not observed in normal individuals and suggests that pAgCC is formed through a more complicated process than arrested callosal development.

A more recent study used high-resolution Q-ball imaging and tractography to map the callosal connectivity in $\mathrm{pAgCC}$ in more detail [42]. The study, with six patients, found considerable variability in both the position and the connectivity of callosal fragments (Fig. 4). Crucially, the cortical connectivity through a given fragment could not be predicted by its position alone; fragments similar in size and position were found to have vastly different connectivity patterns. In addition, a number of heterotopic callosal connections were found between varied cortical areas, extending the asymmetric sigmoid bundle finding of the previous study. These studies indicate that the process of development of callosal fragments in $\mathrm{pAgCC}$ is a complex and plastic process, distinct from normal callosal develop-

Table 1 Summary of imaging and genetic findings. For each of the four congenital malformations discussed, major conventional MRI and diffusion imaging findings are listed, along with genetic findings associated with the condition

\begin{tabular}{|c|c|c|c|}
\hline & Conventional MR & Diffusion imaging & Genetics \\
\hline HGPPS & $\begin{array}{l}\text { 1. Depressed floor of } 4 \text { th ventricle } \\
\text { 2. Hypoplastic pons and medulla, } \\
\text { with "butterfly medulla" sign }\end{array}$ & $\begin{array}{l}\text { 1. Absence of decussating SCP and } \\
\text { pontocerebellar fibers } \\
\text { 2. Ipsilaterally projecting corticospinal tracts }\end{array}$ & ROBO3 mutation [21] \\
\hline PTCD & $\begin{array}{l}\text { 1. Hypoplastic ventral pons } \\
\text { 2. Dorsal pontine cap projecting into } \\
\text { 4th ventricle }\end{array}$ & $\begin{array}{l}\text { 1. Absence of } \mathrm{SCP} \text { and } \mathrm{MCP} \text { decussations } \\
\text { 2. Ectopic dorsal pontine decussating tract }\end{array}$ & $\begin{array}{l}\text { Unknown; no mutations observed } \\
\text { in NTN1 or DCC [28] }\end{array}$ \\
\hline HPE & $\begin{array}{l}\text { 1. Absent ventromedial cerebrum and } \\
\text { anterior hemispheric fissure }\end{array}$ & $\begin{array}{l}\text { 1. CSTs absent in alobar HPE } \\
\text { 2. CST and MCP size correlate with cognitive } \\
\text { performance } \\
\text { 3. Multiple altered supratentorial white matter } \\
\text { tracts in semilobar HPE }\end{array}$ & Multiple associated genes [52] \\
\hline $\mathrm{AgCC}$ & $\begin{array}{l}\text { 1. Callosal absence, with or without } \\
\text { associated cortical malformations } \\
\text { 2. Probst bundles } \\
\text { 3. Reduced VCB tract volume }\end{array}$ & $\begin{array}{l}\text { 1. Heterotopic tracts in partial } \mathrm{AgCC} \\
\text { 2. Reduced FA in VCB }\end{array}$ & Multiple associated genes [35] \\
\hline
\end{tabular}

$H G P P S$ horizontal gaze palsy with progressive scoliosis; $P T C D$ pontine tegmental cap dysplasia; $H P E$ holoprosencephaly; $A g C C$ agenesis of the corpus callosum; $S C P$ superior cerebellar peduncle; $M C P$ middle cerebellar peduncle; $V C B$ ventral cingulum bundle 
ment. It is possible that the same signaling mechanisms that yield aberrant intrahemispheric connectivity (through the Probst bundles) also give rise to novel interhemispheric connectivity patterns through the callosal fragment.

A final study examined the microstructural organization of the cingulum bundle in subjects with $\mathrm{AgCC}$ [43]. Because of the observed clinical deficits in executive function and social-emotional processing that map to the anterior cingulate cortex, it was hypothesized that individuals with $\mathrm{AgCC}$ had reduced organization of the ventral cingulum bundle. DTI tractography was performed in 12 $\mathrm{AgCC}$ patients along with 12 matched controls, and the FA of the ventral cingulum bundle was measured. T1-weighted structural images were also used to measure the tract volume. Compared with controls, the study revealed that $\mathrm{AgCC}$ patients demonstrated a reduced FA specific to the right cingulum bundle, along with bilateral reductions in tract volume. Thus, in addition to the presence of aberrant connections, individuals with $\mathrm{AgCC}$ appear to have alterations to the organization of normal ipsilateral white-matter tracts, which might contribute significantly to the behavioral phenotype. $\mathrm{AgCC}$ might be a more widespread whitematter disorder than previously appreciated.

Table 1 summarizes the classic conventional MR imaging findings, the DTI and HARDI tractographic findings, and the known genetic underpinnings of the four congenital malformations examined in detail in this article.

\section{Future directions}

The same diffusion imaging studies that have advanced our knowledge of congenital brain malformations point to potential clinical uses. In the hindbrain malformations PTCD and HGPPS, while conventional imaging findings can suggest the diagnosis, the DTI findings of defective brainstem midline decussation (HGPPS) and ectopic dorsal transverse pontine fibers (PTCD) are more specific signs. In holoprosencephaly, the size of corticospinal tracts and middle cerebellar peduncles on DTI images correlates well with assessments of neurodevelopment and might be utilized for prognostic purposes.

In studies of $\mathrm{AgCC}$, considerable variation has been found in the connectivity of Probst bundles and, in the case of partial agenesis, callosal fragments. As more patients are examined with diffusion tractography, these variations might be found to correlate with behavioral and cognitive performance. Such findings would be very useful clinically, leading to early prognosis of developmental outcome. Finally, quantitative FA measurements of the cingulum bundle, and likely other white matter structures, represent potentially useful biomarkers for the severity of the condition.

Diffusion imaging has also been applied to more common pediatric conditions that typically do not reveal findings on conventional neuroimaging, such as autism [44-50]. White matter alterations in these neurodevelopmental disorders have been found to be more widespread. As these findings are increasingly correlated with cognitive measures, diffusion imaging might inform cognitive and behavioral therapy on the basis of FA measurements in specific white-matter regions. Also, the combination of quantitative imaging findings with genetic analyses might isolate genes involved in polygenic conditions [51]. Because diffusion imaging provides uniquely detailed quantitative information about white-matter microstructural organization and connectivity, its applications to pediatric neuroradiology are likely to proliferate.

Open Access This article is distributed under the terms of the Creative Commons Attribution Noncommercial License which permits any noncommercial use, distribution, and reproduction in any medium, provided the original author(s) and source are credited.

\section{References}

1. Basser PJ, Mattiello J, Le Bihan D (1994) Estimation of the effective self-diffusion-tensor from the NMR spin echo. J Magn Reson B 103:247-254

2. Pierpaoli C, Jezzard P, Basser PJ et al (1996) Diffusion tensor MR imaging of the human brain. Radiology 201:637-648

3. Stejskal EO, Tanner JE (1965) Spin diffusion measurements: spin echoes in the presence of a time-dependent field gradient. J Chem Phys 42:288-292

4. Mukherjee P, Berman JI, Chung SW et al (2008) Diffusion tensor MR imaging and fiber tractography: theoretic underpinnings. AJNR 29:632-641

5. Beaulieu C (2002) The basis of anisotropic water diffusion in the nervous system - a technical review. NMR Biomed 15:435-455

6. Wakana S, Caprihan A, Panzenboeck MM et al (2007) Reproducibility of quantitative tractography methods applied to cerebral white matter. Neuroimage 36:630-644

7. Charlton RA, Barrick TR, McIntyre DJ (2006) White matter damage on diffusion tensor imaging correlates with age-related cognitive decline. Neurology 66:217-222

8. Niogi SN, Mukherjee P, Ghajar J et al (2008) Extent of microstructural white matter integrity in postconcussive syndrome correlates with impaired cognitive reaction time: a 3T diffusion tensor imaging study of mild traumatic brain injury. AJNR 29:967-973

9. Salo R, Nordahl TE, Buonocore MH et al (2009) Cognitive control and white matter callosal microstructure in methamphetaminedependent subjects: a diffusion tensor imaging study. Biol Psychiatry 65:122-128

10. Schmithorst VJ, Wilke M, Dardzniski BJ et al (2005) Cognitive functions correlated with white matter architecture in a normal pediatric population: a diffusion tensor MRI study. Hum Brain Mapp 26:139-147

11. Skranes J, Vangberg TR, Kulseng S (2007) Clinical findings and white matter abnormalities seen on diffusion tensor imaging in adolescents with very low birth weight. Brain 130:654-666

12. Wozniak JR, Krach L, Mueller BA et al (2007) Neurocognitive and neuroimaging correlates of pediatric traumatic brain injury: a diffusion tensor imaging (DTI) study. Arch Clin Neuropsychol 22:555-568 
13. Conturo TE, Lori NF, Cull TS et al (1999) Tracking neuronal fiber pathways in the living human brain. Proc Natl Acad Sci USA 96:10422-10427

14. Mori S, Kaufmann WE, Pearlson GD et al (1999) Threedimensional tracking of axonal projections in the brain by magnetic resonance imaging. Ann Neurol 45:265-269

15. Wakana S, Jiang H, Nagae-Poetscher LM et al (2004) Fiber tractbased atlas of human white matter anatomy. Radiology 230:77-87

16. Mukherjee P, Berman JI, Chung SW et al (2008) Diffusion tensor MR imaging and fiber tractography: technical considerations. AJNR 29:843-852

17. Ozarslan E, Mareci TH (2003) Generalized diffusion tensor imaging and analytical relationships between diffusion tensor imaging and high angular resolution diffusion imaging. Magn Reson Med 50:955-965

18. Tuch DS (2004) Q-ball imaging. Magn Reson Med 52:1358-1372

19. Hess CP, Mukherjee P, Han ET et al (2006) Q-ball reconstruction of multimodal fiber orientations using the spherical harmonic basis. Magn Reson Med 56:104-117

20. Hess CP, Mukherjee P (2007) Visualizing white matter pathways in the living human brain: diffusion tensor imaging and beyond. Neuroimaging Clin N Am 17:407-426

21. Jen JC, Chan WM, Bosley TM et al (2004) Mutations in a human ROBO gene disrupt hindbrain axon pathway crossing and morphogenesis. Science 304:1509-1513

22. Pieh C, Lengyel D, Neff A et al (2002) Brainstem hypoplasia in familiar horizontal gaze palsy and scoliosis. Neurology 59:462463

23. Rossi A, Catala M, Biancheri R et al (2004) MR imaging of brainstem hypoplasia in horizontal gaze palsy with progressive scoliosis. AJNR 25:1046-1048

24. Sicotte NL, Salamon G, Shattuck DW et al (2006) Diffusion tensor MRI shows abnormal brainstem crossing fibers associated with ROBO3 mutations. Neurology 67:519-521

25. dos Santos AV, Matias S, Saraiva P et al (2006) MR imaging features of brainstem hypoplasia in familial horizontal gaze palsy and scoliosis. AJRN 27:1382-1383

26. Haller S, Wetzel SG, Lütschg J (2008) Functional MRI, DTI and neurophysiology in horizontal gaze palsy with progressive scoliosis. Neuroradiology 50:453-459

27. Jissendi-Tchofo P, Doherty D, McGillivray G et al (2009) Pontine tegmental cap dysplasia: MR imaging and diffusion tensor imaging features of impaired axonal navigation. AJNR 30:113-119

28. Barth PG, Majoie CB, Caan MW et al (2007) Pontine tegmental cap dysplasia: a novel brain malformation with a defect in axonal guidance. Brain 130:2258-2266

29. Barkovich AJ, Simon EM, Clegg NJ et al (2002) Analysis of the cerebral cortex in holoprosencephaly with attention to the sylvian fissures. AJNR 23:143-150

30. Oba H, Barkovich AJ (1995) Holoprosencephaly: an analysis of callosal formation and its relation to development of the interhemispheric fissure. AJNR 16:453-460

31. Simon E, Hevner R, Pinter J et al (2000) Assessment of the deep grey nuclei in holoprosencephaly. AJNR 21:1955-1961

32. Albayram S, Melhem ER, Mori S et al (2002) Holoprosencephaly in children: diffusion tensor MR imaging of white matter tracts of the brainstem - initial experience. Radiology 223:645-651

33. Rollins N (2005) Semilobar holoprosencephaly seen with diffusion tensor imaging and fiber tracking. AJNR 26:21482152
34. Badaruddin DH, Andrews GL, Bolte S et al (2007) Social and behavioral problems of children with agenesis of the corpus callosum. Child Psych and Hum Develop 38:287-302

35. Paul LK, Brown WS, Adolphs R et al (2007) Agenesis of the corpus callosum: genetic, developmental and functional aspects of connectivity. Nat Rev Neurosci 8:287-299

36. Hetts SW, Sherr EH, Chao SD et al (2006) Anomalies of the corpus callosum: an MR analysis of the phenotypic spectrum of associated malformations. AJR 187:1343-1348

37. Lee SK, Mori S, Kim DJ et al (2004) Diffusion tensor MR imaging visualizes the altered hemispheric fiber connection in callosal dysgenesis. AJNR 25:25-28

38. Utsunomiya H, Yamashita S, Takano K et al (2006) Arrangement of fiber tracts forming Probst bundle in complete callosal agenesis: report of two cases with an evaluation by diffusion tensor tractography. Acta Radiol 47:1063-1066

39. Tovar-Moll F, Moll J, de Oliveira-Souza R et al (2007) Neuroplasticity in human callosal dysgenesis: a diffusion tensor imaging study. Cereb Cortex 17:531-541

40. Ren T, Zhang J, Plachez C et al (2007) Diffusion tensor magnetic resonance imaging and tract-tracing analysis of Probst bundle structure in Netrin1- and DCC-deficient mice. J Neurosci 27:10345-10349

41. Alcamo EA, Chirivella L, Dautzenberg M et al (2008) Satb2 regulates callosal projection neuron identity in the developing cerebral cortex. Neuron 57:364-377

42. Wahl M, Strominger Z, Jeremy RJ et al (2009) Variability of homotopic and heterotopic callosal connectivity in partial agenesis of the corpus callosum: a 3T diffusion tensor imaging and Q-ball tractography study. AJNR 30:292-289

43. Nakata Y, Barkovich AJ, Wahl M et al (2009) Diffusion abnormalities and reduced volume of the ventral cingulum bundle in agenesis of the corpus callosum: a $3 \mathrm{~T}$ imaging study. AJNR. doi:10.3174/ajnr.A1527 [Epub ahead of print Feb. 26, 2009]

44. Lee JE, Bigler ED, Alexander AL et al (2007) Diffusion tensor imaging of white matter in the superior temporal gyrus and temporal stem in autism. Neurosci Lett 424:127-132

45. Alexander AL, Lee JE, Lazar M et al (2007) Diffusion tensor imaging of the corpus callosum in autism. Neuroimage 34:61-73

46. Sundaram SK, Kumar A, Makki MI et al (2008) Diffusion tensor imaging of frontal lobe in autism spectrum disorder. Cereb Cortex 18:2659-2665

47. Thomas C, Galia A, Humphreys K et al (2009) Reduced structural connectivity in ventral visual cortex in congenital prosopagnosia. Nat Neurosci 12:29-31

48. Berhmann M, Avidan G, Gao F et al (2007) Structural imaging reveals anatomical variations in inferotemporal cortex in congenital prosopagnosia. Cereb Cortex 17:2354-2363

49. Avidan G, Hasson U, Malach R et al (2005) Detailed exploration of face-related processing in congenital prosopagnosia: 2. Functional neuroimaging findings. J Cogn Neurosci 17:1150-1167

50. Rouw R, Scholte SH (2007) Increased structural connectivity in grapheme-color synesthesia. Nat Neurosci 10:792-797

51. Kempf L, Nicodemus KK, Kolachana B et al (2008) Functional polymorphisms in PRODH are associated with risk and protection for schizophrenia and fronto-striatal structure and function. PLoS Genet 4:e1000252

52. Oliver G, Geng X (1999) Pathogenesis of holoprosencephaly. J Clin Invest 119:1403-1413 